\title{
A systematic review of primary Sjögren's syndrome in male and paediatric populations
}

\author{
Simrun Virdee ${ }^{1} \cdot$ James Greenan-Barrett $^{1} \cdot$ Coziana Ciurtin $^{2}$ (I)
}

Received: 12 May 2017 /Revised: 26 June 2017 / Accepted: 28 June 2017 / Published online: 22 July 2017

(C) The Author(s) 2017. This article is an open access publication

\begin{abstract}
Primary Sjögren's syndrome (pSS) is a chronic multisystem autoimmune rheumatic disease characterised by female predominance. Although the disease is rare in the male and paediatric populations, it has been suggested that it may have a different disease phenotype, which has not been investigated before using a systematic approach. A systematic literature search of PubMed databases (updated to December 2016) was performed to identify all published data on the epidemiological, clinical and laboratory manifestations of pSS in the male and paediatric populations. The literature search of the male and paediatric pSS studies identified 2025 and 186 citations, respectively, out of which 7 and 5 fulfilled our inclusion criteria and were analysed further. The range of age at disease onset was 9.4-10.7 years for children and 39.456.9 years at diagnosis for male patients. We identified a prevalence of extra-glandular manifestations between 52.6-92.3\% in the male population and $50.0-84.6 \%$ in children, while abnormal sialometry was only reported in the paediatric population, with a prevalence between 71.4 and $81.8 \%$. There was a significant variation of positive serological markers, with anti-Ro antibodies reported between $15.7-75.0 \%$ and 36.4 $84.6 \%$, and anti-La antibodies between $5.6-51.7 \%$ and $27.3-$
\end{abstract}

Simrun Virdee and James Greenan-Barrett equally contributed to this paper.

Electronic supplementary material The online version of this article (doi:10.1007/s10067-017-3745-z) contains supplementary material, which is available to authorized users.

Coziana Ciurtin

c.ciurtin@ucl.ac.uk

1 University College London Medical School, London, UK

2 Department of Rheumatology, University College London, 250 Euston Road, London NW1 2PG, UK
$65.4 \%$, in the male and paediatric populations, respectively. The characteristics of pSS in the male and paediatric populations varied according to different studies. When compared to data available from $\mathrm{pSS}$ adult populations, children diagnosed with pSS reported less dryness and had a higher prevalence of parotitis, lymphadenopathy and systemic symptoms and male patients were younger at the time of diagnosis. This systematic review contributes to a better understanding of the epidemiology of pSS in rare populations. Large longitudinal cohort studies comparing male with female patients and adult with paediatric patients are needed.

Keywords Primary Sjögren's syndrome $\cdot$ Male patients · Children · Systematic review

\section{Introduction}

Primary Sjögren's syndrome (pSS) is a chronic multisystem autoimmune rheumatic disease, associated in the majority of cases with infiltration of exocrine glands, particularly the salivary and lacrimal glands, by lymphocytes and plasma cells, resulting in xerostomia and xerophthalmia. pSS is a heterogeneous disease; clinical manifestations range from localised glandular disease to complex and even life-threatening systemic features affecting the neurological, renal, musculoskeletal, dermatological, haematological, and pulmonary systems. This autoimmune rheumatic disease is $9-20$ times more common in females than males according to different studies [1-4], and although commonest between the ages of 30 and 60 , it is also described in younger and older age groups [5]. There is a similar female predominance in the paediatric population (F:M = 5-7:1) [6, 7]. The prevalence of pSS in the general population ranged in Greece from 0.09 to $0.23 \%$ [4, 8], in 
the UK from 0.14 to $1.60 \%$ [9] and in Slovenia was estimated at $0.60 \%$ [10].

In 2016, newly updated classification criteria were published by the American College of Rheumatology/European League Against Rheumatism [11, 12]. The proposed classification criteria weigh 5 criteria items with a score of either 1 or 3 and a total score of at least $4 / 9$, in the absence of any exclusion criteria, is required for a diagnosis. These weighted criteria improve on the previous American-European Consensus Group (AECG) and American College of Rheumatology (ACR) classification criteria by ensuring that a positive anti-SSA/Ro or salivary gland biopsy is required for a diagnosis. Along with other changes, in the absence of anti$\mathrm{SSA} / \mathrm{Ro}$, anti-SSB/La is no longer considered a criteria item.

\section{Methods}

\section{Search strategy}

We performed a systematic literature search of PubMed, covering papers published between January 1984 and December 2016. In search of the articles on the male population with Sjögren's syndrome, we used the following MeSH terms: 'Sjögren's syndrome' and 'men' OR 'male'. To identify relevant publications on the paediatric population, we used 'Sjögren's syndrome' and 'child' OR 'paediatrics' as MeSH terms. The automatically selected papers were manually screened by reading the titles and abstracts. Both searches were limited to human studies and English language publications but no limitations were based on the country of origin or ethnicity of patients. Following the selection of articles that met the inclusion criteria, their references were reviewed for any relevant papers that had been missed by the PubMed search, and relevant studies were added to the final analysis.

\section{Study selection}

The study selection was carried out independently by two authors (SV and JGB) based on the inclusion criteria mentioned above, and subsequently compared-any discrepancies were discussed with the third author (CC) and a consensus was reached. The general exclusion criteria applied by authors were the following: incorrect patient groups (female cohort, secondary Sj gren's syndrome, patients selected based on specific comorbidities), incorrect study types (case reports, interventional studies, editorials, commentaries, surveys and questionnaires), studies that analysed therapeutics, quality of life or cost-benefit analysis and genetic, laboratory or pathology studies. The full texts of the remaining articles were read and the above criteria were further applied.

The data from the eligible articles was then extracted and analysed. The study selection process is detailed in Fig. 1. One article (retrieved as a reference in another paper) was excluded from the final analysis, as despite our attempts to contact the author, the full text could not be retrieved [13].

\section{Data extraction}

We extracted data on epidemiological (study type, number of patients, country of origin, etc.), clinical and laboratory features and presented it in four tables. Table 1 details the epidemiological data on both male and paediatric populations. In Table 2, we included information related to the clinical features associated with pSS in both populations. The laboratory data were organised into two tables (Tables 3 and 4) as the data set analysed differed between the male and paediatric population. Any data reported as percentages were converted to actual number of patients to allow us to calculate ranges of data across all the studies analysed.

\section{Assessment of study quality}

We used a critical appraisal tool (The Joanna Briggs Institute Prevalence Critical Appraisal Tool) designed for use in systematic reviews addressing questions of prevalence to assess the quality of our studies [14]. The appraisal tool comprises nine assessment questions related to the quality of studies including the population representativeness, recruitment strategy, sample size, description of study subjects, use of objective, standard criteria for subject classification, quality of outcome measurements, appropriate statistical analysis, identification of confounding factors and identification of subpopulations, where appropriate. These criteria were assessed as present, absent, unclear or not applicable, as per the methodological guidance published for this critical appraisal tool [15]. The assessment was completed by all three authors independently and a consensus was reached for every study. Because of the low prevalence of $\mathrm{pSS}$ in the paediatric and male populations, the majority of the studies evaluated cohorts from secondary and tertiary centres and used various classification criteria (justified by the lack of classification criteria in the paediatric population, and various revisions of classification criteria for adult $\mathrm{pSS}$ over time). In addition, the measurement of different clinical manifestations was not standardised and it relied on medical records and expert opinion. The data analysis did not have significant coverage of the identified sample as, in the majority of studies, the 
Fig. 1 a Flowchart of study selection for papers referring to the pSS male population. $\mathbf{b}$ Flowchart of study selection for papers referring to $\mathrm{pSS}$ paediatric populations a

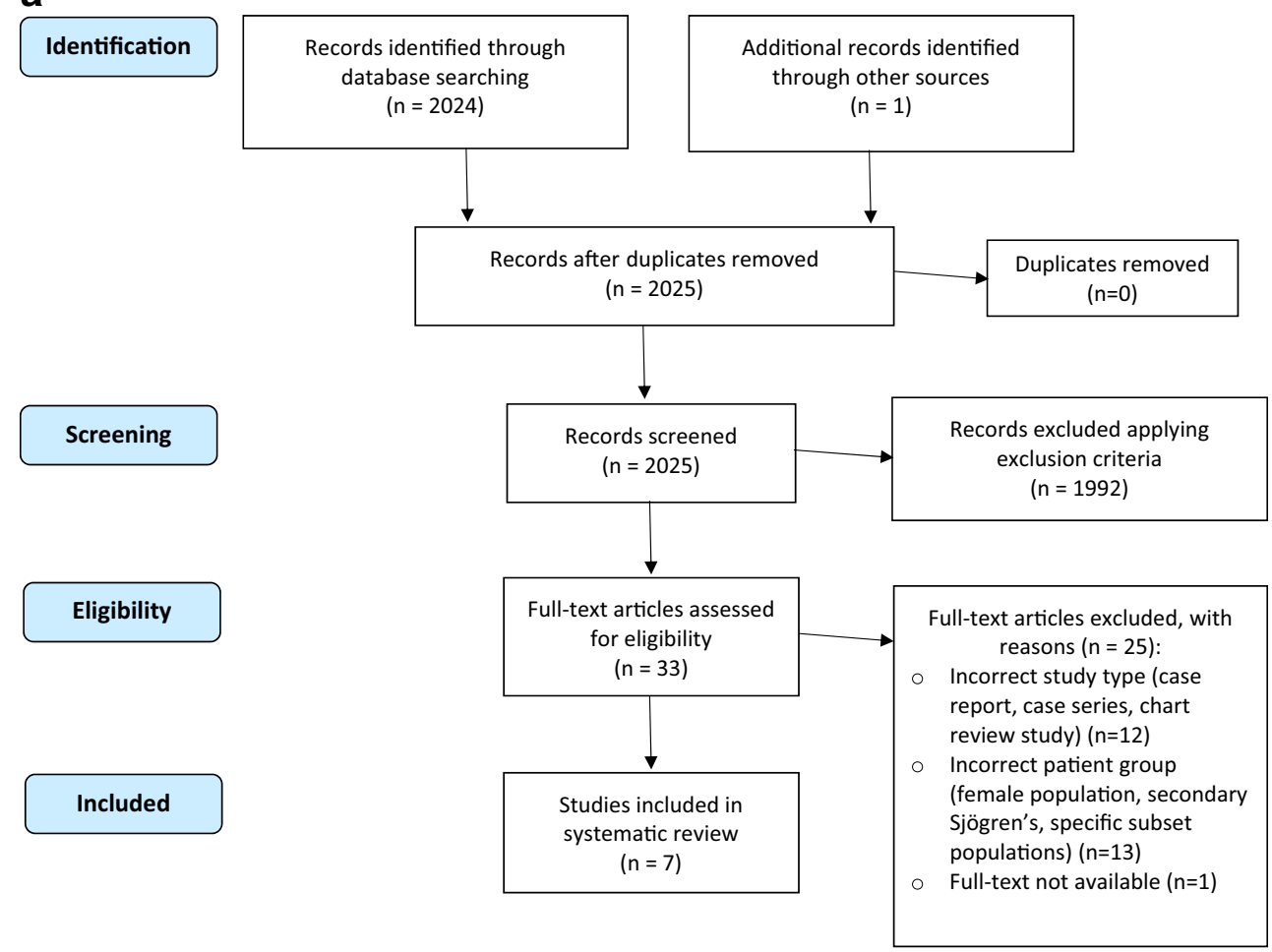

b

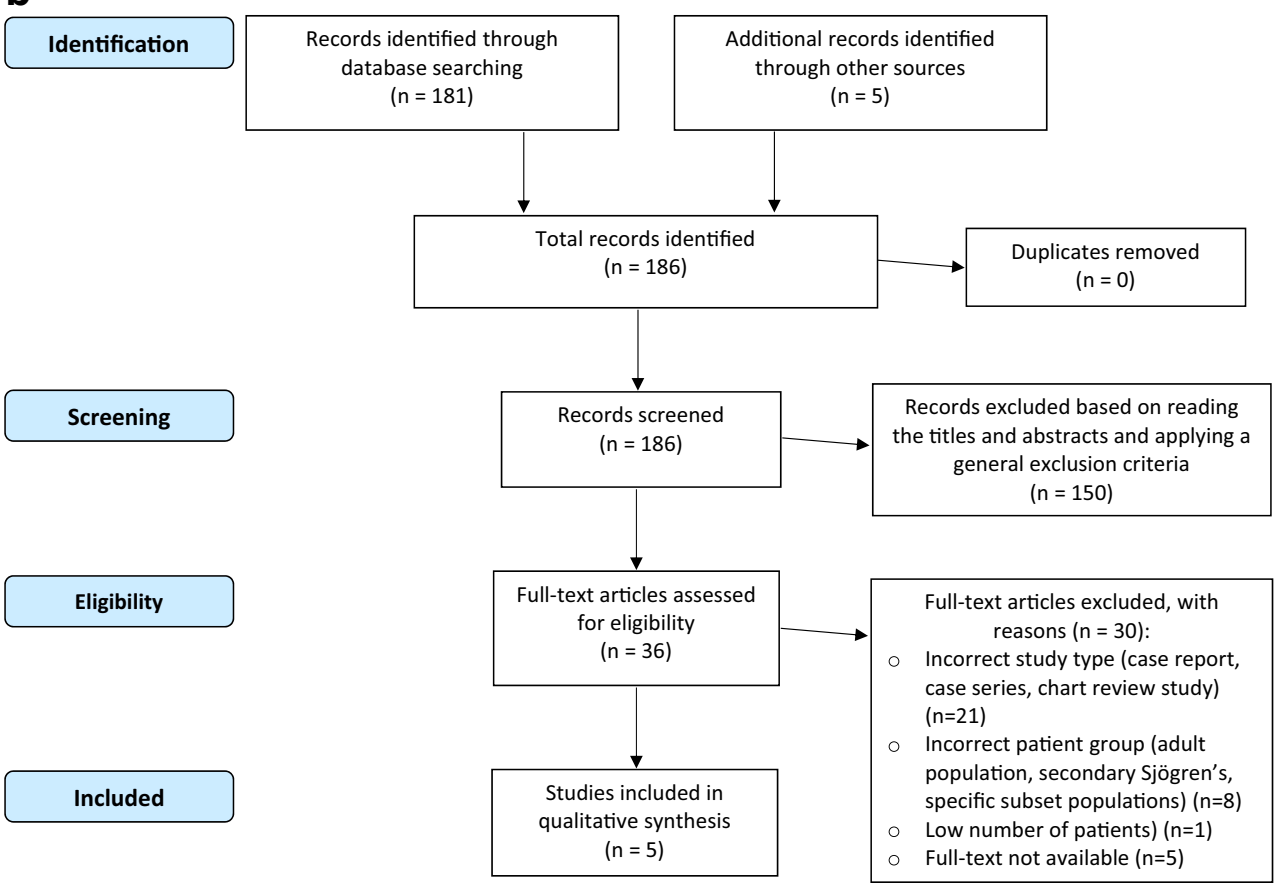

paediatric cases were not compared with adult patients, and the male case was pre-selected and compared to consecutive female case series from a different cohort.

A compromise was made by the authors to include papers which used different classification criteria for pSS because of the small number of studies available in the male and paediatric populations. Consequently, and in addition to the lack of fulfilment of the majority of the nine questions included in the appraisal checklist and because of the high study heterogeneity, we could not calculate reliable pooled prevalence rates for different disease manifestations and perform a meta-analysis. 


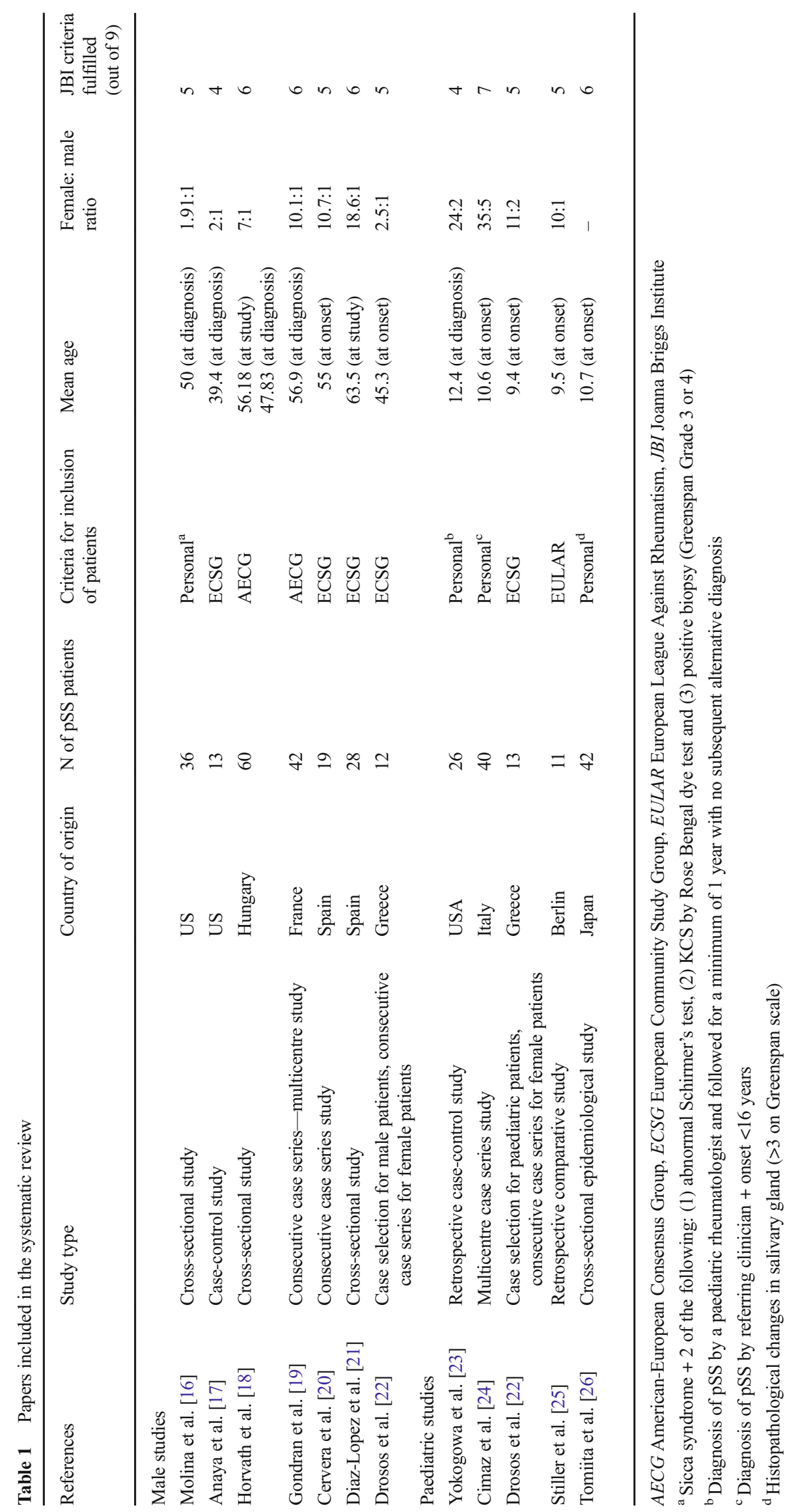




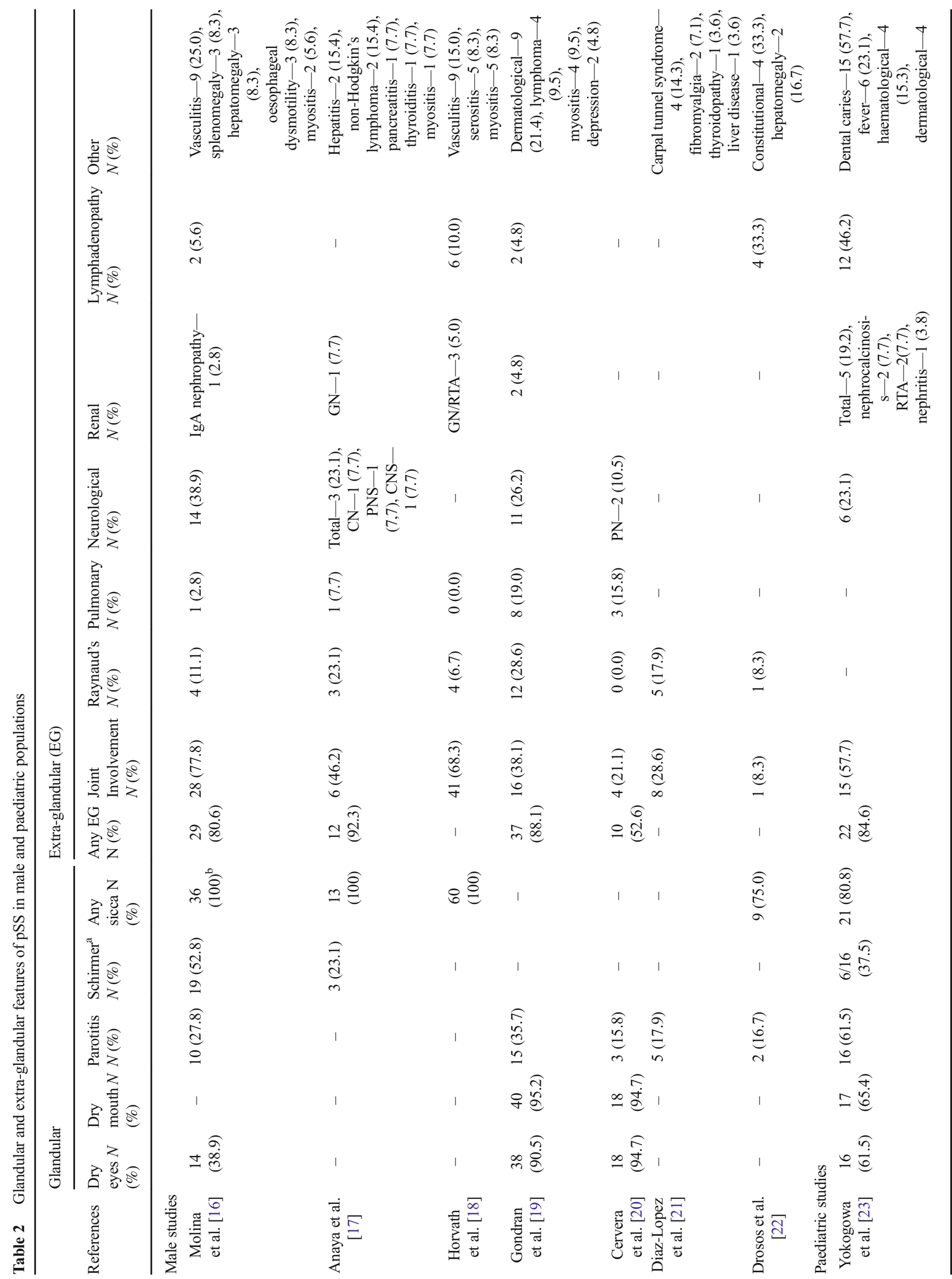




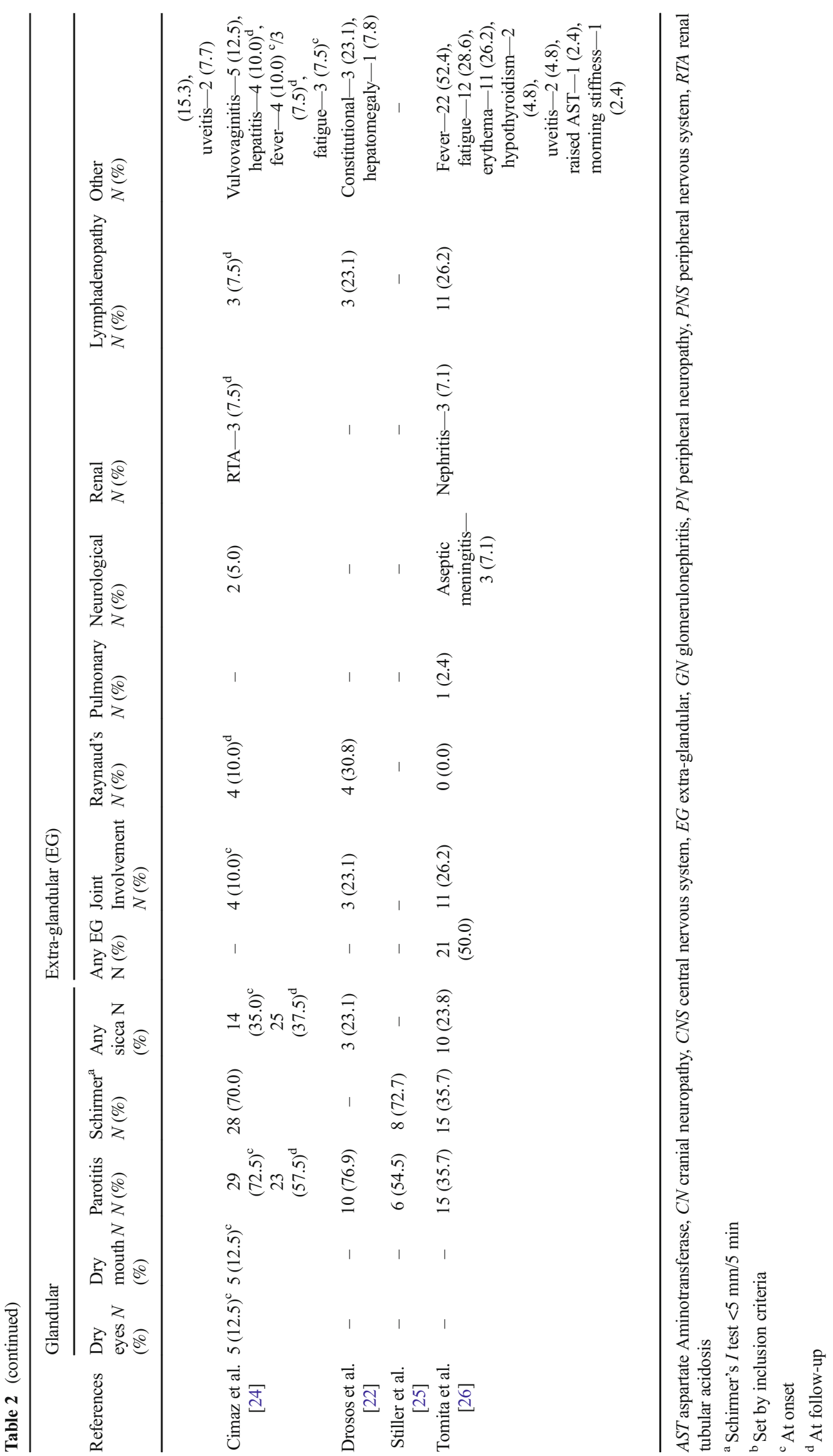




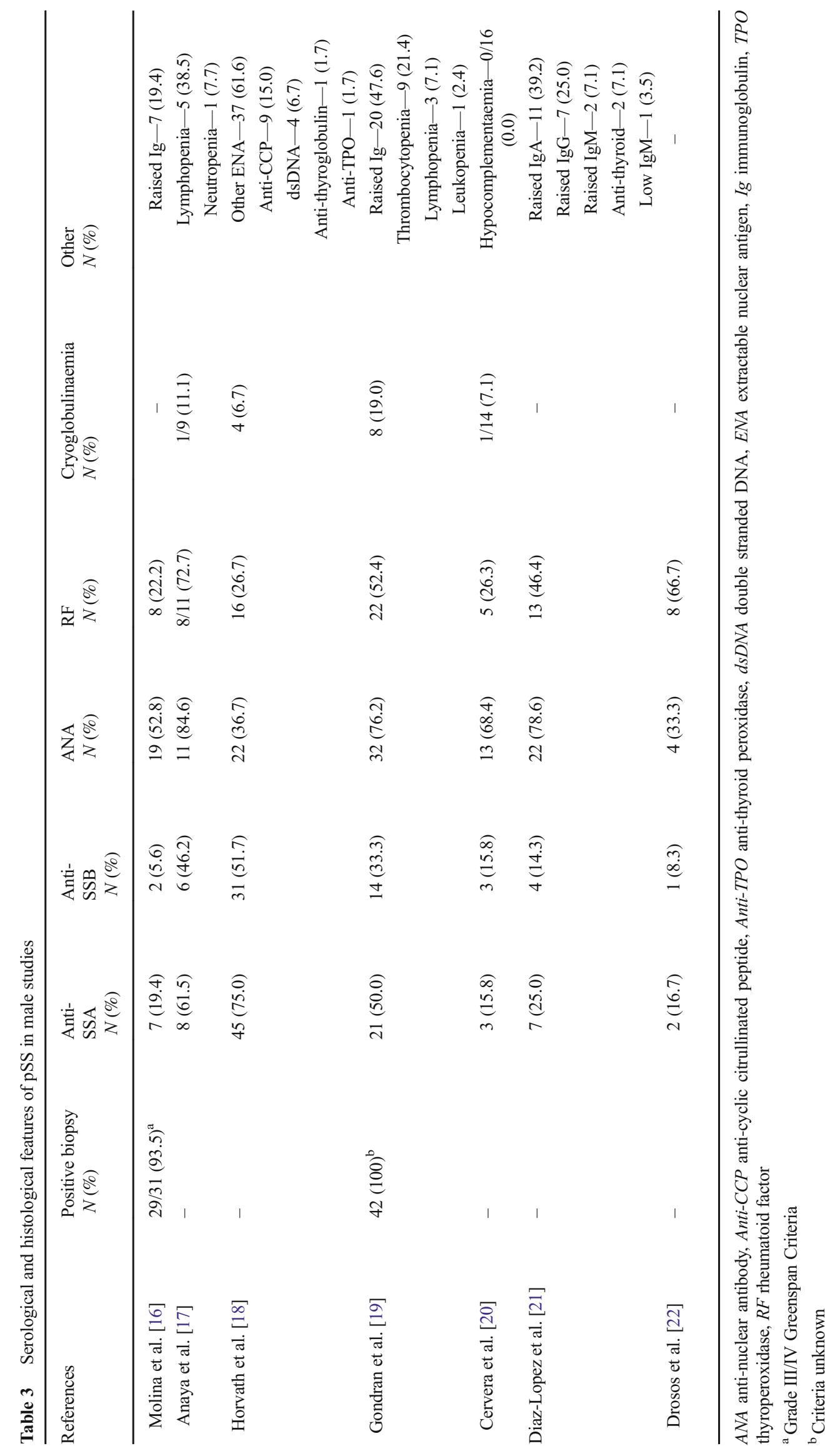




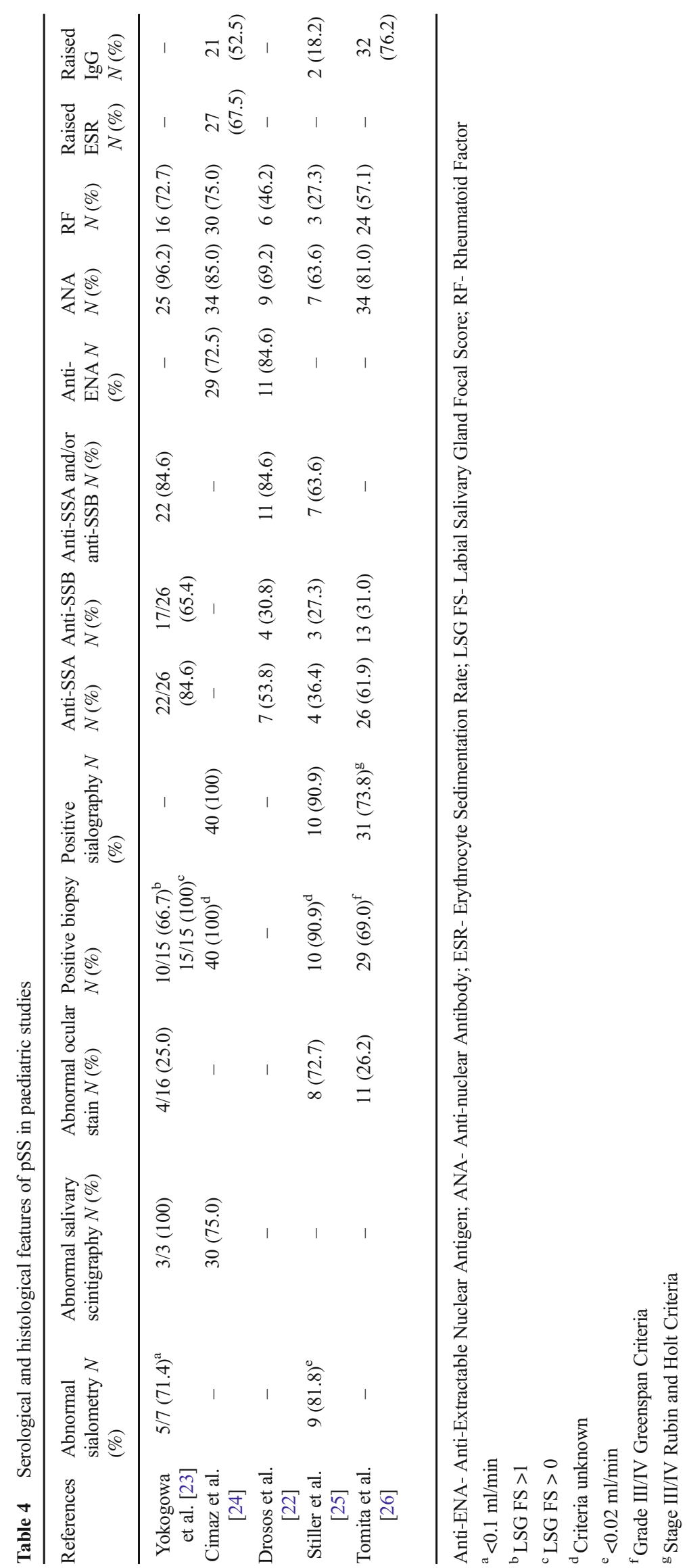




\section{Results}

\section{Demographics/epidemiological}

After assessment of study quality, we included data from seven studies on the male population [16-22] and five on the paediatric population [22-26]; this data is presented in Table 1 . The total adult male cohort included in our systematic review consisted of 210 patients (range 12-60 patients/per study). The paediatric cohort consisted of 132 patients (range 11-42 patients/per study). All of the male papers included comparisons with female patient population, while in the paediatric studies, only one study compared children with adult pSS population [23]. Three studies reported on patients from the American population, eight were European and one was Japanese. The criteria for patient inclusion varied. In the male studies, four papers used the European Community Study Group (ECSG) classification [27], two papers used the 2002 AECG [28] and one paper used its own personal criteria (Table 1). One paediatric study used the ECSG criteria, one used the European League Against Rheumatism (EULAR) criteria [29] and the other three studies used different personal criteria. The mean age of the patients was available for all studies. However, three different data sets were described: age at onset, age at presentation and age at diagnosis. Four out of the five paediatric papers included in our final analysis quoted age at onset (range 9.5-10.7 years). Across the cohort of paediatric patients, where reported, the female:male ratio was $8: 1$.

\section{Clinical features}

The data regarding clinical features are presented in Table 2.

\section{Glandular features}

Data about the prevalence of parotitis were available in 10 out of 12 studies. A total of 35 adult male and 76 paediatric patients presented with parotitis as a manifestation of pSS (range 15.8-35.7\% and 35.7-76.9\%, respectively). All studies reported on dryness, although this was classified as 'any sicca' symptoms in some studies and as 'dry eyes' and 'dry mouth' in others. In the four male studies that reported sicca symptoms, these were present in $75.0-100 \%$ of patients. In the four paediatric studies reporting dryness, the subjective sicca symptoms were present in $23.1-80.8 \%$ of patients.

\section{Extra-glandular features}

The presence of any extra-glandular features was described in four papers reporting on the male population and in two of the paediatric papers. A proportion of $52.6-92.3 \%$ of male patients and $50.0-84.6 \%$ of paediatric patients had these manifestations. Joint involvement was the most commonly ascertained data set (all studies, apart from Stiller et al. 2000 ( [25]) reported on it). Joint involvement, defined as arthritis or arthralgia, was reported in $8.3-77.8 \%$ of male patients and $10.0-57.7 \%$ of paediatric patients. Only two papers reported separately on arthritis and arthralgia in male population with pSS, arthralgia being found in 18/28 and 5/6 of patients with joint involvement, respectively $[16,17]$. Raynaud's, pulmonary and neurological involvement were reported in both the male $(8.3-28.6 \%, 0.0-19.8 \%$ and $10.5-38.9 \%$, respectively) and paediatric populations $(0.0-30.8 \%, 2.4 \%$ and $5.0-23.1 \%$, respectively). Renal involvement and lymphadenopathy were found in children in the following proportions in different studies: $7.1-19.2 \%$ and $7.5-46.2 \%$, respectively, while the frequency of these symptoms was lower in the male population (2.8-7.7\% and 4.8-33.3\%, respectively). Many other extra-glandular features were occasionally reported; of note, there was a high prevalence of fever $(52.4 \%)$ and dental caries (57.7\%) reported by two paediatric studies [23, 26].

\section{Laboratory features}

Due to variation of the laboratory features reported between the male and paediatric studies, the data has been presented separately (in Tables 3 and 4). With the exception of one paper, which reported on the presence of anti-extractable nuclear antigen (ENA) antibodies [24], all papers were reported on the core serology - the presence of anti-SSA (Ro), antiSSB (La), anti-nuclear antibodies (ANA) and rheumatoid factor (RF). Anti-SSA, anti-SSB, ANA and RF were positive in $15.7-75.0 \%, 5.6-51.7 \%, 33.3-84.6 \%, 22.2-72.7 \%$, respectively, in the male population, and $36.4-84.6 \%, 27.3-65.4 \%$, $63.6-96.2 \%, 27.3-75.0 \%$, respectively, in the paediatric population, suggesting a high variability between different studies. Cryoglobulinaemia was only reported in the male population (in four out of seven studies) with a prevalence of 6.7$19.0 \%$. A number of other serological markers were occasionally reported (Tables 3 and 4).

Data on non-serological investigations were available for the paediatric population and included pathological biopsy, sialometry, scintigraphy, ocular stains and sialography. In the male population, only salivary gland biopsy data were retrievable from the papers included in our analysis, with a proportion of $93.5-100 \%$ patients having a positive biopsy. In the paediatric population, a pathological biopsy ranged from 66.7 to $100 \%$; two out of the four studies described no criteria for defining the histological criteria used for pSS classification.

In Table 5 (supplementary information), we summarised the main differences between male and female populations, and paediatric and adult populations as reported by the studies included in our analysis; however, comparisons between this systematic review and data available from other systematic 
reviews in adult pSS populations were beyond the purpose of this paper.

\section{Discussion}

Although pSS is an autoimmune disease with wellrecognised female predomination, a proportion of up to $10 \%$ of adult patients diagnosed with this condition are male. In the paediatric population, the female to male ratio is 6-7:1 [24, 30]. The diagnosis of pSS in children is delayed, in many cases, because children less frequently report dryness due to their good saliva and tear reserve and frequently present with extraglandular clinical features suggestive of other autoimmune diseases [31]. Although the disease burden is significant in both male and paediatric populations, good quality studies are lacking. To our knowledge, this is the first attempt to review systematically the literature on male and paediatric populations with pSS.

Many of the studies we selected based on our inclusion criteria involved the selection of relevant paediatric or male cases from large cohorts.

The classification criteria for pSS were revised several times in the last few years and again very recently [11, 12]; therefore, different inclusion criteria were used in different studies. The main difference between the ECSG [29] and AECG [28] classification criteria is that the AECG criteria require the presence of positive biopsy or serology tests to classify patients as having pSS. In addition, the AECG criteria also mention the need to exclude mimicking pathology, such as head and neck radiation treatment, hepatitis $\mathrm{C}$ and use of anticholinergic drugs.

We have taken into consideration that a comparison between studies including patients classified in different ways might lead to erroneous conclusions; therefore, we presented the results as intervals of proportions of patients reported as having different disease manifestations. A pooled prevalence meta-analysis could not be performed because of the high heterogeneity of the patient population, which would have led to unreliable conclusions.

A significant proportion of studies (5/12) included a low number of patients (less than 20). Although all the male studies also evaluated a certain number of female patients, the male cases were pre-selected (because of their rarity) and compared to a variable number of consecutive female cases (therefore, all the studies were affected by patient selection bias).

One of the other limitations of this systematic analysis was the selection of paediatric studies which relied on expert opinion for diagnosis, which is the consequence of lack of specific classification criteria for paediatric pSS. It is recognised that the available classification criteria designed for adult pSS population have significant limitations when used in children [32].

One of the main conclusions of this systematic analysis was that parotid gland involvement was common in children with pSS despite being recognised that, with the exception of mumps parotitis, all other causes of parotitis in children are rare [33]. Parotitis is therefore recognised as a characteristic disease feature in paediatric pSS [31]. Sicca symptoms were less common in children for the reasons discussed above. Symptoms of dryness are recognised to be more common in the adult population affected by pSS because of the correlation between the destruction of the acinar cells and age [34, 35]. Despite this, the symptoms of eye dryness were reported with a high variability in the male studies, with a prevalence range of 38.9-94.7\%.

We also found that in the paediatric population, constitutional symptoms and lymphadenopathy were prevalent, which can be explained by a particular disease phenotype in children or other confounders (frequent associated viral infection during childhood, parental vigilance in detecting high temperature, etc.). However, lymphadenopathy despite being generally less commonly reported in the adult population, it was found as more common in male than female patients in only one study [18]. The joint involvement in adult pSS, partly explained by the disease phenotype and partly by the agerelated associated joint pathology, showed no specific trend in the male versus female population, as the studies included in our analysis reported inconsistent data ( [18], [20], [22]).

There was a significant variation of positive serological markers in both the male and paediatric populations. As the quality of studies and patient classification criteria used were very heterogeneous, we cannot infer any reliable comparison with the reported positive serology prevalence from $\mathrm{pSS}$ adult studies [36]. The trend towards a higher positive serology prevalence in children can be explained again by the difficulty to diagnose pSS in children with reasonably well-preserved exocrine gland secretion without specific serology, and could explain the need for additional investigations to facilitate the diagnosis (we found that invasive investigations, such as scintigraphy, sialometry and biopsy were more frequently performed in paediatric studies). It was previously identified that the presence of antibodies directed against the Ro/La ribonucleoprotein complexes have been correlated with younger age, more severe dysfunction of the exocrine glands and a higher prevalence of extra-glandular manifestations in the adult population [36]. Our systematic analysis identified a prevalence of extra-glandular manifestations between $52.6-92.3 \%$ in the male population and $50.0-84.6 \%$ in children, while abnormal sialometry was reported only in the paediatric population, with a prevalence between 71.4 and $81.8 \%$.

A case series of children with pSS reported that although the serological markers were not relevant for diagnosis, all patients had specific lymphocytic infiltration of labial salivary 
glands and sialectasis [37]; subsequently, the authors concluded that salivary and lachrymal gland histopathology in this age group is highly recommended for accurate diagnosis.

Previous research comparing pSS patients with early and late disease onset found no significant differences between their clinical presentation and incidence of diagnostic test positivity; however, the early onset of pSS was defined as diagnosis before the age of 40 and the study did not include children [5]. A recent systematic review and meta-analysis of pSS epidemiology reported a female/male ratio in prevalence rate for pSS of 10.72:1, without providing details about the differences in clinical presentation or laboratory features of pSS in female compared to male patients [38].

In summary, this systematic review found that children diagnosed with pSS reported less frequently symptoms of dryness, and had a higher prevalence of systemic symptoms, including fever and lymphadenopathy, as well as parotitis, in comparison to adult populations [39, 40].

Male patients with pSS were younger at the time of diagnosis than the pooled aged at diagnosis in the general pSS adult population, as estimated by a recent systematic review and meta-analysis [38]; however, male patients did not have any consistently different clinical or serological features when compared to female pSS patients in different studies, which can be explained by different pSS classification criteria used, patient selection bias or true heterogeneity of the male pSS population.

Our systematic review highlighted the difficulties related to data collection in rare populations. In addition, the different clinical presentation and absence of validated classification criteria for the paediatric population with pSS in comparison to the adult population makes reliable diagnosis very difficult and highly dependent on expert opinion.

We also found that despite the increased clinician interest in defining different disease phenotypes, such as male and paediatric pSS, all the studies available came from developed countries and their conclusions are difficult to extrapolate.

In conclusion, our systematic review highlighted the most commonly reported clinical manifestations and serological markers of pSS in male and children population, raising clinicians' awareness about particular disease features in populations rarely affected by pSS. This could have significant implications in facilitating the diagnosis of pSS in male and children. Future work, including large longitudinal cohort studies comparing male versus female patients, and adult versus paediatric patients, will enable us to differentiate reliably the disease presentation and evolution in these selected categories of patients.

\section{Significance and innovation}

- This is the first systematic review of pSS in rare populations
- Large, multicentre, good-quality studies of pSS in rare populations and classification criteria for $\mathrm{pSS}$ in paediatric population are lacking

- Children with pSS have less dryness, and more frequently experienced systemic symptoms and parotid enlargement

- There were no consistent differential features of male pSS patients when compared to adult female patient population

\section{Compliance with ethical standards}

\section{Disclosures None.}

Open Access This article is distributed under the terms of the Creative Commons Attribution 4.0 International License (http:// creativecommons.org/licenses/by/4.0/), which permits unrestricted use, distribution, and reproduction in any medium, provided you give appropriate credit to the original author(s) and the source, provide a link to the Creative Commons license, and indicate if changes were made.

\section{References}

1. Pillemer SR, Matteson EL, Jacobsson LT, Martens PB, Melton LJ 3rd, O'Fallon WM et al (2001) Incidence of physician-diagnosed primary Sjogren syndrome in residents of Olmsted County, Minnesota. Mayo Clin Proc 76:593-599

2. Plesivcnik Novljan M, Rozman B, Hocevar A, Grmek M, Kveder $\mathrm{T}$, Tomsic M (2001) Incidence of primary Sjogren's syndrome in Slovenia. Ann Rheum Dis 63:874-876

3. Haugen AJ, Peen E, Hulten B, Johannessen AC, Brun JG, Halse AK et al (2008) Estimation of the prevalence of primary Sjogren's syndrome in two age-different community-based populations using two sets of classification criteria: the Hordaland Health Study. Scand J Rheumatol 37:30-34

4. Anagnostopoulos I, Zinzaras E, Alexiou I, Papathanasiou AA, Davas E, Koutroumpas A et al (2010) The prevalence of rheumatic diseases in central Greece: a population survey. BMC Musculoskelet Disord 11:98

5. Botsios C, Furlan A, Ostuni P, Sfriso P, Andretta M, Ometto F et al (2011) Elderly onset of primary Sjogren's syndrome: clinical manifestations, serological features and oral/ocular diagnostic tests. Comparison with adult and young onset of the disease in a cohort of 336 Italian patients. Joint Bone Spine 78:171-174

6. Kassan SS, Moutsopoulos HM (2004) Clinical manifestations and early diagnosis of Sjogren syndrome. Arch Intern Med 164:12751284

7. de Souza TR, Silva IH, Carvalho AT, Gomes VB, Duarte AP, Leao JC et al (2012) Juvenile Sjogren syndrome: distinctive age, unique findings. Pediatr Dent 34:427-430

8. Dafni UG, Tzioufas AG, Staikos P, Skopouli FN, Moutsopoulos HM (1997) Prevalence of Sjogren's syndrome in a closed rural community. Ann Rheum Dis 56:521-525

9. Bowman SJ, Ibrahim GH, Holmes G, Hamburger J, Ainsworth JR (2004) Estimating the prevalence among Caucasian women of primary Sjogren's syndrome in two general practices in Birmingham, UK. Scand J Rheumatol 33:39-43

10. Tomsic M, Logar D, Grmek M, Perkovic T, Kveder T (1999) Prevalence of Sjogren's syndrome in Slovenia. Rheumatology (Oxford) 38:164-170

11. Shiboski CH, Shiboski SC, Seror R, Criswell LA, Labetoulle M, Lietman TM et al (2017) 2016 American College of Rheumatology/ 
European League Against Rheumatism classification criteria for primary Sjogren's syndrome: a consensus and data-driven methodology involving three international patient cohorts. Ann Rheum Dis 76:9-16

12. Shiboski CH, Shiboski SC, Seror R, Criswell LA, Labetoulle M, Lietman TM et al (2017) 2016 American College of Rheumatology/European League Against Rheumatism classification criteria for primary Sjogren's syndrome: a consensus and data-driven methodology involving three international patient cohorts. Arthritis Rheum 69(1):35-45

13. Brennan MTFP (1999) Sex differences in primary Sjögren's syndrome. J Rheumatol 26(11):2373-2376

14. Munn Z, Moola S, Riitano D, Lisy K (2014) The development of a critical appraisal tool for use in systematic reviews addressing questions of prevalence. Int J Health Policy Manag 3(3):123-128

15. Munn Z, Moola S, Lisy K, Riitano D, Tufanaru C (2015) Methodological guidance for systematic reviews of observational epidemiological studies reporting prevalence and cumulative incidence data. Int J Evid Based Healthc 13(3):147-153

16. Molina R, Provost TT, Arnett FC, Bias WB, Hochberg MC, Wilson RW et al (1986) Primary Sjogren's syndrome in men. Clinical, serologic, and immunogenetic features. Am J Med 80(1):23-31

17. Anaya JM, Liu GT, D'Souza E, Ogawa N, Luan X, Talal N (1995) Primary Sjogren's syndrome in men. Ann Rheum Dis 54(9):748751

18. Horvath IF, Szodoray P, Zeher M (2008) Primary Sjogren's syndrome in men: clinical and immunological characteristic based on a large cohort of Hungarian patients. Clin Rheumatol 27(12):14791483

19. Gondran G, Fauchais A, Lambert M, Ly K, Launay D, Queyrel V et al (2008) Primary Sjogren's syndrome in men. Scand J Rheumatol 37(4):300-305

20. Cervera R, Font J, Ramos-Casals M, Garcia-Carrasco M, Rosas J, Morla RM et al (2000) Primary Sjogren's syndrome in men: clinical and immunological characteristics. Lupus 9(1):61-64

21. Diaz-Lopez C, Geli C, Corominas H, Malat N, Diaz-Torner C, Llobet JM et al (2004) Are there clinical or serological differences between male and female patients with primary Sjogren's syndrome? J Rheumatol 31(7):1352-1355

22. Drosos AA, Tsiakou EK, Tsifetaki N, Politi EN, SiamopoulouMavridou A (1997) Subgroups of primary Sjogren's syndrome. Sjogren's syndrome in male and paediatric Greek patients. Ann Rheum Dis 56(5):333-335

23. Yokogawa N, Lieberman SM, Sherry DD, Vivino FB (2016) Features of childhood Sjogren's syndrome in comparison to adult Sjogren's syndrome: considerations in establishing child-specific diagnostic criteria. Clin Exp Rheumatol 34(2):343-351

24. Cimaz R, Casadei A, Rose C, Bartunkova J, Sediva A, Falcini F et al (2003) Primary Sjogren syndrome in the paediatric age: a multicentre survey. Eur J Pediatr 162(10):661-665

25. Stiller M, Golder W, Doring E, Biedermann T (2000) Primary and secondary Sjogren's syndrome in children - a comparative study. Clin Oral Investig 4(3):176-182
26. Tomiita M, Saito K, Kohno Y, Shimojo N, Fujikawa S, Niimi H (1997) The clinical features of Sjogren's syndrome in Japanese children. Acta Paediatr Jpn 39(2):268-272

27. Vitali C, Bombardieri S, Moutsopoulos HM, Balestrieri G, Bencivelli W, Bernstein RM et al (1993) Preliminary criteria for the classification of Sjogren's syndrome. Results of a prospective concerted action supported by the European Community. Arthritis Rheum 36(3):340-347

28. Vitali C, Bombardieri S, Jonsson R, Moutsopoulos HM, Alexander EL, Carsons SE et al (2002) Classification criteria for Sjogren's syndrome: a revised version of the European criteria proposed by the American-European Consensus Group. Ann Rheum Dis 61(6): 554-558

29. Vitali C, Moutsopoulos HM, Bombardieri S (1994) The European Community Study Group on diagnostic criteria for Sjogren's syndrome. Sensitivity and specificity of tests for ocular and oral involvement in Sjogren's syndrome. Ann Rheum Dis 53(10):637647

30. Civilibal M, Canpolat N, Yurt A, Kurugoglu S, Erdamar S, Bagci O et al (2007) A child with primary Sjogren syndrome and a review of the literature. Clin Pediatr (Phila) 46(8):738-742

31. Singer NG, Tomanova-Soltys I, Lowe R (2008) Sjogren's syndrome in childhood. Curr Rheumatol Rep 10(2):147-155

32. Houghton K, Malleson P, Cabral D, Petty R, Tucker L (2005) Primary Sjogren's syndrome in children and adolescents: are proposed diagnostic criteria applicable? J Rheumatol 32(11):22252232

33. Ellies M, Laskawi R (2010) Diseases of the salivary glands in infants and adolescents. Head Face Med 6:1

34. Baum BJ, Ship JA, Wu AJ (1992) Salivary gland function and aging: a model for studying the interaction of aging and systemic disease. Crit Rev Oral Biol Med 4(1):53-64

35. Van Haeringen NJ (1997) Aging and the lacrimal system. Br J Ophthalmol 81(10):824-826

36. Bournia VK, Vlachoyiannopoulos PG (2012) Subgroups of Sjogren syndrome patients according to serological profiles. J Autoimmun 39(1-2):15-26

37. Saad Magalhaes C, de Souza Medeiros PB, Oliveira-Sato J, Custodio-Domingues MA (2011) Clinical presentation and salivary gland histopathology of paediatric primary Sjogren's syndrome. Clin Exp Rheumatol 29(3):589-593

38. Qin B, Wang J, Yang Z, Yang M, Ma N, Huang F et al (2015) Epidemiology of primary Sjogren's syndrome: a systematic review and meta-analysis. Ann Rheum Dis 74(11):1983-1989

39. Ramos-Casals M, Brito-Zeron P, Seror R, Bootsma H, Bowman SJ, Dorner $\mathrm{T}$ et al (2015) Characterization of systemic disease in primary Sjogren's syndrome: EULAR-SS Task Force recommendations for articular, cutaneous, pulmonary and renal involvements. Rheumatology (Oxford) 54(12):2230-2238

40. Epstein JB, Villines DC, Sroussi HY (2015) Oral symptoms and oral function in people with Sjogren's syndrome. Clin Exp Rheumatol 33(1):132-133 